\title{
Effect of Nutrient Management and Planting Geometry on Productivity of Hybrid Rice (Oryza sativa L.) Cultivars
}

\author{
Ranjita Bezbaruha, Ravi Chandra Sharma, Pabitra Banik* \\ Agricultural and Ecological Research Unit, Indian Statistical Institute, Kolkata, India. \\ Email: ${ }^{*}$ pbanik@isical.ac.in
}

Received December $29^{\text {th }}, 2010$; revised May $13^{\text {th }}, 2011$; accepted July $14^{\text {th }}, 2011$.

\begin{abstract}
Field experiments were conducted during the wet seasons of 2006 and 2007 at the Agricultural Experimental Farm of the Indian Statistical Institute, Giridih, a part of eastern plateau region of India. The study was designed to investigate the effect of planting geometry and nutrient management practices on productivity of two hybrid rice cultivars. Split-plot design with three replications was adopted to carry out the experiment by allocating combinations of treatments of planting geometry and rice cultivar in main-plots and nutrient management treatments in sub-plots. "CNRH-3" rice proved its efficiency in terms of grain yield that was also reflected in yield attributing characters such as number of productive tillers, number of grains per panicle, length of panicle, panicle weight, test weight and harvest index. Higher rice grain yield was registered when the cultivars grown in $20 \mathrm{~cm} \times 20 \mathrm{~cm}$ planting geometry. Rice cultivars grown with the application of inorganic fertilizers alone produced maximum grain yield and also recorded higher values of ancillary characters. The maximum amount of $N, P$ and $K$ was taken up by the "CNRH-3" rice, whereas maximum residual soil fertility was recorded in "Pro Agro 6201" rice. Maximum N, P and K uptake values were recorded in $20 \mathrm{~cm} \times 20 \mathrm{~cm}$ crop geometry and inorganic fertilizers treatment.
\end{abstract}

Keywords: Hybrid Rice Cultivars, Planting Geometry, Vermicompost, Nutrient Uptake, Soil Fertility

\section{Introduction}

One of every three peoples depends on rice for more than half of their daily food and one in nine (approximately 700 million) depends on rainfed rice. Ninety percent of the world's rice is grown and consumed in Asia. Rice is also an important staple food in some countries of Latin America and Africa. Asian rice production has increased by 24\% during 1965 to 1980 and that was attributed to the use of higher rate of fertilizers, mainly $\mathrm{N}$-fertilizer. Rice productivity is now at stagnant situation or declining in areas where N-fertilizer application is very high; it has also raised the concerns about sustainability of monoculture rice [1]. Food security in India (1.6 billion by 2050 that will require $450 \mathrm{Mt}$ of food grain production) is a challenge [2]. To achieve food security, hybrid rice can be one of the most feasible options to increase $15 \%$ to $20 \%$ of food production $[3,4]$. The hybrid cultivars are more responsive to higher doses of nutrients $[5,6]$ and thereby the yield potentiality is all high.

It is a big concern that whether the agronomic practices, especially planting geometry for hybrid rice are same as for conventional rice. Thus, there is a need to optimize the planting geometry for hybrid rice [7]. Proper planting geometry have more advantages such as, to maximize light utilization efficiency, improves aeration within crop canopy, enhances soil respiration and provides better weed control thereby higher crop yields [8].

Of late, there has been serious concern about longterm adverse effect of continuous and indiscriminate use of inorganic fertilizers on soil health, biodiversity and environment [9]. The organic matter in sub-tropics soils is low because of high temperature and intense microbial activity. Soil organic matter is the key attribute of soil quality [10] therefore organic matter has to be replenished to maintain the soil health. Apart from nutritional effects [11], application of organic manure influences plant physiologically. It also provides growth regulating substances to plants and modifies soil physical behaviour [12]. Vermicompost is a good source of organic manure that can be used as an alternative to chemical fertilizers in rice cultivation [13]. Organic matter dynamics is similar in different cropping systems but its significance for 
specific soil properties or crop productivity varies considerably with soil type [14,15]. With the above back drop the experiments have been undertaken to measure the effect of crop geometry and the usefulness of organic materials on yield of low land hybrid rice and soil fertility.

\section{Materials and Methods}

\subsection{Study Site}

A study was carried out at the Agricultural Experimental Farm of the Indian Statistical Institute, Giridih (at $24^{\circ} 1{ }^{\prime} \mathrm{N}$, $86^{\circ} 3^{\prime} \mathrm{E}$ and altitude $\left.920^{\prime}\right)$, India during the wet seasons of 2006 and 2007. The average annual rainfall of the study area is $1343 \mathrm{~mm}$ but the distribution is highly seasonal (about $86 \%$ of total rainfall occurs in between June to September). The average maximum and minimum temperatures are $23.8^{\circ} \mathrm{C}$ and $12.6^{\circ} \mathrm{C}$ respectively. Average annual potential evapotranspiration is $1293 \mathrm{~mm}$ with the relative humidity ranges from $78 \%$ to $95 \%$. The soil was moderately well drained lateritic sandy loam (30.0\% coarse sand, $26.8 \%$ fine sand, $25.0 \%$ silt and $18.2 \%$ clay). Soil was slightly acidic in reaction (6.4) with low in organic carbon (0.52\%), available $\mathrm{N}\left(132 \mathrm{~kg} \mathrm{~N} \mathrm{ha}^{-1}\right)$ and $\mathrm{P}$ (12 kg P ha ${ }^{-1}$ ) but medium in K (156 kg K ha $\left.{ }^{-1}\right)$.

\subsection{Experimental Setup}

The experiment was laid out in split-plot design and replicated thrice. Combinations of rice cultivars $\left(\mathrm{V}_{1}\right.$ : $\mathrm{CNRH}$ 3 and $V_{2}$ : Pro Agro-6201) and crop geometry $\left(G_{1}: 15\right.$ $\mathrm{cm} \times 15 \mathrm{~cm}, \mathrm{G}_{2}: 20 \mathrm{~cm} \times 20 \mathrm{~cm}$ and $\left.\mathrm{G}_{3}: 25 \mathrm{~cm} \times 25 \mathrm{~cm}\right)$ were allocated in the main-plots and nutrient management practices such as $\mathrm{F}_{0}$ : absolute control, $\mathrm{F}_{1}$ : recommended dose (RD, 140:60:60 kg N, $\mathrm{P}_{2} \mathrm{O}_{5}, \mathrm{~K}_{2} \mathrm{O}$ ha $^{-1}$ ) through inorganic sources, $\mathrm{F}_{2}$ : $\mathrm{RD}$ of $\mathrm{N}$ through vermicompost, $\mathrm{F}_{3}: 50 \% \mathrm{RD}$ of NPK through inorganic $+50 \%$ $\mathrm{RD}$ of $\mathrm{N}$ through vermicompost and $\mathrm{F}_{4}: 75 \% \mathrm{RD}$ of NPK through inorganic $+25 \%$ through vermicompost were assigned in sub-plots. The recommended dose of NPK was applied in the form of urea (46-0-0), single super phosphate (0-16-0) and muriate of potash (0-0-60). Vermicompost (1.25-0.8-0.65) was incorporated in soil as per the treatment at the time of final ploughing. Rice cultivars were transplanted on $15^{\text {th }}$ July and $18^{\text {th }}$ July and were harvested on $9^{\text {th }}$ December and $11^{\text {th }}$ December in 2006 and 2007, respectively. Agronomic management practices and plant protection measures were followed as per the recommendation.

\subsection{Soil Sampling and Analysis}

Soil samples were collected from each plot at the depth of $0-20 \mathrm{~cm}$ just after harvest of rice in both the years. These soil samples were sieved $(2 \mathrm{~mm})$ and analyzed for available $\mathrm{N}$ by alkaline potassium permanganate method [16] and organic carbon by wet oxidation method [17]. Mineralizable $\mathrm{P}$ and exchangeable $\mathrm{K}$ were estimated by Olsen's method [18] and neutral normal ammonium acetate method [19], respectively. Concentrations of N, P and $\mathrm{K}$ in rice grain and straw were estimated by using the standard methods as advocated by Jackson [19].

\subsection{Statistical Analysis}

The data obtained during the study were subjected to statistical analysis using the IRRISTAT (software developed by International Rice Research Institute, Philippines).

\section{Results}

\subsection{Plant Height}

Plant height of rice cultivars was significantly influenced by the crop geometry and nutrient management practices (Table 1). Plant height of "CNRH 3" rice was higher $(88.66 \mathrm{~cm})$ over the Pro Agro $6201(85.88 \mathrm{~cm})$. Rice grown at $15 \mathrm{~cm} \times 15 \mathrm{~cm}$ apart recorded higher plant height $(88.42 \mathrm{~cm})$ whereas $25 \mathrm{~cm} \times 25 \mathrm{~cm}$ spacing recorded shortest ones $(86.16 \mathrm{~cm})$. Rice grown with $100 \%$ RD of NPK supplied through inorganic sources $\left(F_{1}\right)$ produced tallest plants but it was statistically at par with that of the $\mathrm{F}_{4}$ treatment.

\subsection{Productive Tillers}

"CNRH 3" rice produced maximum numbers of reproductive tillers $\left(349 \mathrm{~m}^{-2}\right)$ (Table 1). Rice grown at $20 \mathrm{~cm}$ $\times 20 \mathrm{~cm}$ spacing, irrespective of cultivars and fertilizer treatments, produced highest reproductive tillers per unit area $\left(395 \mathrm{~m}^{-2}\right)$ and which was followed by $25 \mathrm{~cm} \times 25$ $\mathrm{cm}$ treatment. Among the nutrient management practices, $F_{1}$ treatment was recorded $46 \%$ higher reproductive tillers over the $\mathrm{F}_{0}$ treatment.

\subsection{Filled Grains per Panicle}

“CNRH 3" rice produced 31\% higher filled grains per panicle over Pro Agro 6201 (Table 1). Rice transplanted in $20 \mathrm{~cm} \times 20 \mathrm{~cm}$ spacing produced maximum number of filled grains per panicle (73.13) followed by rice when grown at $25 \mathrm{~cm} \times 25 \mathrm{~cm}$ spacing (64.38). Rice had 85\% and $63 \%$ higher grains per panicle when grown with $F_{1}$ and $\mathrm{F}_{4}$ treatments respectively over the $\mathrm{F}_{0}$.

\subsection{Panicle Length and Weight}

Both panicle length $(21.65 \mathrm{~cm})$ and weight $(2.55 \mathrm{~g})$ were recorded maximum in "CNRH 3" rice (Table 1). Irrespective of cultivars, both the values were higher when rice was grown at $20 \mathrm{~cm} \times 20 \mathrm{~cm}$ apart followed by at 25 $\mathrm{cm} \times 25 \mathrm{~cm}$ spacing. Fertilizer treatment $\mathrm{F}_{1}$ had 65\% higher panicle length over the control $\left(\mathrm{F}_{0}\right)$. 


\subsection{Test Weight}

Rice cultivar CNRH 3 (26.24 g) recorded higher test weight (weight of 1000 grains) over the other one (Table 1). Significantly highest test weight $(26.50 \mathrm{~g})$ of rice was registered when grown in $20 \mathrm{~cm} \times 20 \mathrm{~cm}$ crop spacing whereas least test weight was in $15 \mathrm{~cm} \times 15 \mathrm{~cm}$. Test weight was significantly affected by the nutrient management practices. Fertilizer treatment $F_{1}$ followed by $F_{4}$ recorded the higher test weight over the others.

\subsection{Grain Yield}

The "CNRH 3" rice produced the highest grain yield $\left(4527 \mathrm{~kg} \cdot \mathrm{ha}^{-1}\right.$ ) and harvest index (HI; 0.47) over Pro Agro-6201 (Table 2). Maximum grain yield was recorded when rice cultivars were transplanted in $20 \mathrm{~cm} \times$ $20 \mathrm{~cm}$ crop spacing ( $4804 \mathrm{~kg} \cdot \mathrm{ha}^{-1}$ ) followed by $25 \mathrm{~cm} \times$ $25 \mathrm{~cm}$ spacing. HI also recorded the similar trend. There was a significant variation in grain yield and $\mathrm{HI}$ due to nutrient management practices as well and were registered highest when the cultivars grown with the $F_{1}$. Whereas least values of grain yield and HI were recorded in the $\mathrm{F}_{0}$.

\subsection{Nutrient Uptake}

Among the rice cultivars, higher $\mathrm{N}, \mathrm{P}$ and $\mathrm{K}$ uptakes were recorded by CNRH3 (Table 3). Cultivars grown at $20 \mathrm{~cm} \times 20 \mathrm{~cm}$ spacing accumulated higher nutrients while least amount of nutrients uptake was at $15 \mathrm{~cm} \times 15$ $\mathrm{cm}$. Rice cultivars recorded maximum nutrients (NPK) uptake when they received $100 \%$ nutrients through inorganic fertilizers $\left(\mathrm{F}_{2}\right)$ but it was statistically at par with the treatment $\mathrm{F}_{4}$. However, least amount of nutrients uptake was found when rice grown without any fertilizers $\left(\mathrm{F}_{0}\right)$.

\subsection{Residual Soil Nutrients}

Residual soil nutrients ( $\mathrm{N}, \mathrm{P}$ and $\mathrm{K}$ ) values were maximum in "Pro Agro 6201" rice (Table 4). Crop geometry did not have significant effect on soil fertility. Although the values were maximum in $15 \mathrm{~cm} \times 15 \mathrm{~cm}$ crop spacing. The VC treatment $\left(\mathrm{F}_{2}\right)$ had maximum residual soil nutrients (NPK) values whereas these were least in $F_{0}$ followed by $\mathrm{F}_{1}$ treatments.

Table 1. Effect of crop geometry and nutrient management practices on plant growth and yield attributes of hybrid rice cultivars (pooled data of 2006 and 2007).

\begin{tabular}{|c|c|c|c|c|c|c|}
\hline Treatment & $\begin{array}{l}\text { Plant height } \\
\quad \text { (cm) }\end{array}$ & $\begin{array}{l}\text { Productive } \\
\text { Tillers } \mathrm{m}^{-2}\end{array}$ & $\begin{array}{l}\text { Filled grains } \\
\text { panicle }^{-1}\end{array}$ & $\begin{array}{l}\text { Panicle length } \\
\text { (cm) }\end{array}$ & $\begin{array}{l}\text { Panicle weight } \\
\text { (g) }\end{array}$ & $\begin{array}{l}\text { Test weight } \\
\text { (g) }\end{array}$ \\
\hline \multicolumn{7}{|l|}{ Cultivars } \\
\hline $\mathrm{V}_{1}$ : CNRH3 & 88.66 & 348.99 & 74.42 & 21.65 & 2.55 & 26.24 \\
\hline $\mathrm{V}_{2}$ : Pro Agro 6201 & 85.88 & 334.31 & 56.58 & 20.42 & 2.14 & 25.66 \\
\hline $\operatorname{SEm} \pm$ & 0.95 & 5.39 & 7.28 & 0.22 & 0.08 & 0.16 \\
\hline $\operatorname{LSD}(p=0.05)$ & 2.11 & 12.00 & 16.23 & 0.49 & 0.18 & 0.35 \\
\hline \multicolumn{7}{|l|}{ Crop geometry } \\
\hline $\mathrm{G}_{1}: 15 \mathrm{~cm} \times 15 \mathrm{~cm}$ & 88.42 & 287.26 & 59.59 & 20.85 & 1.92 & 25.78 \\
\hline $\mathrm{G}_{2}: 20 \mathrm{~cm} \times 20 \mathrm{~cm}$ & 86.16 & 394.73 & 73.13 & 21.50 & 2.69 & 26.50 \\
\hline $\mathrm{G}_{3}: 25 \mathrm{~cm} \times 25 \mathrm{~cm}$ & 87.05 & 343.33 & 64.38 & 20.80 & 2.38 & 25.52 \\
\hline $\mathrm{SEm} \pm$ & 0.19 & 7.03 & 1.93 & 0.08 & 0.09 & 0.23 \\
\hline $\operatorname{LSD}(p=0.05)$ & 0.42 & 15.66 & 4.30 & 0.18 & 0.20 & 0.51 \\
\hline \multicolumn{7}{|l|}{ Nutrient } \\
\hline $\mathrm{F}_{0}$ : Control & 80.92 & 272.09 & 45.09 & 15.67 & 1.90 & 22.59 \\
\hline $\mathrm{F}_{1}: \operatorname{RDF}(160: 60: 60)$ & 92.23 & 398.69 & 83.49 & 25.86 & 2.85 & 28.07 \\
\hline $\mathrm{F}_{2}: 100 \% \mathrm{RDF}$ through VC* & 84.79 & 319.90 & 59.72 & 19.98 & 2.10 & 24.01 \\
\hline $\mathrm{F}_{3}: 50 \% \mathrm{RDF}+50 \% \mathrm{VC}$ & 87.52 & 342.78 & 67.52 & 20.67 & 2.19 & 25.12 \\
\hline $\mathrm{F}_{4}: 75 \% \mathrm{RDF}+25 \% \mathrm{VC}$ & 90.63 & 376.96 & 73.32 & 23.03 & 2.56 & 26.94 \\
\hline $\operatorname{SEm} \pm$ & 1.60 & 16.46 & 5.14 & 0.98 & 0.16 & 0.42 \\
\hline $\operatorname{LSD}(p=0.05)$ & 3.22 & 33.12 & 10.34 & 1.97 & 0.33 & 0.85 \\
\hline
\end{tabular}

*Vermicompost. 
Table 2. Grain yield, straw yield and harvesting index as influenced by rice cultivars, crop geometry and nutrient management practices.

\begin{tabular}{|c|c|c|c|c|c|c|}
\hline \multirow{2}{*}{ Treatment } & \multicolumn{2}{|c|}{ Grain yield $\left(\mathrm{kg} \cdot \mathrm{ha}^{-1}\right)$} & \multicolumn{2}{|c|}{ Straw yield $\left(\mathrm{kg} \cdot \mathrm{ha}^{-1}\right)$} & \multicolumn{2}{|c|}{ Harvesting index } \\
\hline & 2006 & 2007 & 2006 & 2007 & 2006 & 2007 \\
\hline \multicolumn{7}{|l|}{ Cultivars } \\
\hline $\mathrm{V}_{1}$ : CNRH3 & 4577 & 4869 & 5266 & 5506 & 0.47 & 0.47 \\
\hline $\mathrm{V}_{2}$ : Pro Agro 6201 & 4415 & 4683 & 5440 & 5695 & 0.45 & 0.45 \\
\hline $\operatorname{SEm} \pm$ & 55 & 50 & 69 & 60 & & \\
\hline $\operatorname{LSD}(p=0.05)$ & 123 & 112 & 153 & 134 & & \\
\hline \multicolumn{7}{|l|}{ Crop geometry } \\
\hline $\mathrm{G}_{1}: 15 \mathrm{~cm} \times 15 \mathrm{~cm}$ & 4195 & 4485 & 5339 & 5482 & 0.44 & 0.45 \\
\hline $\mathrm{G}_{2}: 20 \mathrm{~cm} \times 20 \mathrm{~cm}$ & 4804 & 5091 & 5446 & 5741 & 0.47 & 0.47 \\
\hline $\mathrm{G}_{3}: 25 \mathrm{~cm} \times 25 \mathrm{~cm}$ & 4493 & 4756 & 5274 & 5583 & 0.46 & 0.46 \\
\hline $\operatorname{SEm} \pm$ & 87 & 76 & 35 & 57 & & \\
\hline $\operatorname{LSD}(p=0.05)$ & 194 & 169 & 77 & 126 & & \\
\hline \multicolumn{7}{|l|}{ Nutrient } \\
\hline $\mathrm{F}_{0}$ : Control & 3928 & 4035 & 5424 & 5702 & 0.42 & 0.42 \\
\hline $\mathrm{F}_{1}: \mathrm{RDF}(160: 60: 60)$ & 4992 & 5203 & 5517 & 5595 & 0.48 & 0.48 \\
\hline $\mathrm{F}_{2}: 100 \% \mathrm{RDF}$ through $\mathrm{VC}^{*}$ & 4298 & 4637 & 5127 & 5314 & 0.46 & 0.47 \\
\hline $\mathrm{F}_{3}: 50 \% \mathrm{RDF}+50 \% \mathrm{VC}$ & 4485 & 4849 & 5308 & 5579 & 0.46 & 0.47 \\
\hline $\mathrm{F}_{4}: 75 \% \mathrm{RDF}+25 \% \mathrm{VC}$ & 4773 & 5158 & 5382 & 5831 & 0.47 & 0.47 \\
\hline SEm \pm & 66 & 58 & 46 & 57 & & \\
\hline $\operatorname{LSD}(p=0.05)$ & 132 & 117 & 93 & 114 & & \\
\hline
\end{tabular}

*Vermicompost.

Table 3. Nutrients (NPK) uptake as influenced by rice cultivars, crop geometry and nutrient management practices.

\begin{tabular}{|c|c|c|c|c|c|c|}
\hline \multirow{3}{*}{$\begin{array}{ll} & \text { Treatment } \\
\text { Cultivars } & \end{array}$} & \multicolumn{6}{|c|}{ Nutrients uptake $\left(\mathrm{kg} \cdot \mathrm{ha}^{-1}\right)$} \\
\hline & \multicolumn{2}{|c|}{ Nitrogen } & \multicolumn{2}{|c|}{ Phosphorus } & \multicolumn{2}{|c|}{ Potassium } \\
\hline & 2006 & 2007 & 2006 & 2007 & 2006 & 2007 \\
\hline $\mathrm{V}_{1}:$ CNRH3 & 86.52 & 89.66 & 18.87 & 21.08 & 128.95 & 133.15 \\
\hline $\mathrm{V}_{2}$ : Pro Agro 6201 & 84.81 & 87.35 & 17.09 & 20.28 & 123.91 & 129.07 \\
\hline $\operatorname{SEm} \pm$ & 0.57 & 0.44 & 0.55 & 0.35 & 1.85 & 1.44 \\
\hline $\operatorname{LSD}(p=0.05)$ & 1.26 & 0.98 & 1.23 & 0.78 & 4.12 & 3.21 \\
\hline \multicolumn{7}{|l|}{ Crop geometry } \\
\hline $\mathrm{G}_{1}: 15 \mathrm{~cm} \times 15 \mathrm{~cm}$ & 81.97 & 83.76 & 14.98 & 17.41 & 116.84 & 121.15 \\
\hline $\mathrm{G}_{2}: 20 \mathrm{~cm} \times 20 \mathrm{~cm}$ & 92.48 & 93.99 & 20.12 & 23.63 & 133.89 & 139.69 \\
\hline $\mathrm{G}_{3}: 25 \mathrm{~cm} \times 25 \mathrm{~cm}$ & 85.21 & 87.83 & 18.86 & 21.03 & 128.49 & 132.48 \\
\hline $\operatorname{SEm} \pm$ & 1.44 & 1.21 & 0.85 & 0.72 & 2.03 & 1.50 \\
\hline $\operatorname{LSD}(p=0.05)$ & 3.20 & 2.70 & 1.90 & 1.60 & 4.53 & 3.35 \\
\hline \multicolumn{7}{|l|}{ Nutrient } \\
\hline $\mathrm{F}_{0}$ : Control & 70.79 & 71.23 & 13.53 & 15.84 & 109.53 & 112.35 \\
\hline $\mathrm{F}_{1}: \mathrm{RDF}(160: 60: 60)$ & 100.73 & 104.07 & 23.59 & 25.89 & 151.27 & 156.24 \\
\hline $\mathrm{F}_{2}: 100 \% \mathrm{RDF}$ through $\mathrm{VC} *$ & 79.38 & 81.14 & 15.47 & 17.91 & 117.79 & 120.47 \\
\hline $\mathrm{F}_{3}: 50 \% \mathrm{RDF}+50 \% \mathrm{VC}$ & 87.81 & 90.02 & 17.79 & 20.83 & 121.69 & 127.98 \\
\hline $\mathrm{F}_{4}: 75 \% \mathrm{RDF}+25 \% \mathrm{VC}$ & 93.99 & 96.21 & 19.53 & 23.97 & 131.85 & 138.54 \\
\hline $\operatorname{SEm} \pm$ & 2.75 & 2.09 & 1.16 & 1.08 & 3.10 & 2.80 \\
\hline $\operatorname{LSD}(p=0.05)$ & 5.54 & 4.21 & 2.33 & 2.17 & 6.23 & 5.64 \\
\hline
\end{tabular}

*Vermicompost. 
Table 4. Residual soil nutrients status as influenced by rice cultivars, crop geometry and nutrient management practices.

\begin{tabular}{|c|c|c|c|c|c|c|}
\hline \multirow{3}{*}{$\begin{array}{ll} & \text { Treatment } \\
& \\
\text { Cultivars } & \end{array}$} & \multicolumn{6}{|c|}{ Residual soil nutrients status $\left(\mathrm{kg} \cdot \mathrm{ha}^{-1}\right)$} \\
\hline & \multicolumn{2}{|c|}{ Nitrogen } & \multicolumn{2}{|c|}{ Phosphorus } & \multicolumn{2}{|c|}{ Potassium } \\
\hline & 2006 & 2007 & 2006 & 2007 & 2006 & 2007 \\
\hline $\mathrm{V}_{1}$ : CNRH3 & 141.08 & 146.21 & 12.15 & 13.53 & 123.87 & 129.24 \\
\hline $\mathrm{V}_{2}$ : Pro Agro 6201 & 145.78 & 149.52 & 13.38 & 14.43 & 125.57 & 132.08 \\
\hline $\mathrm{SEm} \pm$ & 1.14 & 0.96 & 0.30 & 0.23 & 0.84 & 0.80 \\
\hline $\operatorname{LSD}(p=0.05)$ & 2.54 & 2.14 & 0.67 & 0.52 & 1.87 & 1.79 \\
\hline \multicolumn{7}{|l|}{ Crop geometry } \\
\hline $\mathrm{G}_{1}: 15 \mathrm{~cm} \times 15 \mathrm{~cm}$ & 148.23 & 152.86 & 13.91 & 15.12 & 128.87 & 134.28 \\
\hline $\mathrm{G}_{2}: 20 \mathrm{~cm} \times 20 \mathrm{~cm}$ & 139.57 & 143.94 & 11.87 & 12.96 & 121.88 & 127.95 \\
\hline $\mathrm{G}_{3}: 25 \mathrm{~cm} \times 25 \mathrm{~cm}$ & 142.48 & 146.87 & 12.51 & 13.85 & 123.42 & 129.74 \\
\hline $\operatorname{SEm} \pm$ & 1.06 & 0.66 & 0.50 & 0.44 & 1.81 & 1.50 \\
\hline $\operatorname{LSD}(p=0.05)$ & 2.36 & 1.48 & 1.12 & 0.98 & 4.03 & 3.35 \\
\hline \multicolumn{7}{|l|}{ Nutrient } \\
\hline $\mathrm{F}_{0}$ : Control & 136.98 & 137.97 & 10.09 & 10.94 & 109.56 & 111.75 \\
\hline $\mathrm{F}_{1}: \mathrm{RDF}(160: 60: 60)$ & 141.60 & 144.53 & 11.45 & 12.97 & 122.32 & 127.47 \\
\hline $\mathrm{F}_{2}: 100 \% \mathrm{RDF}$ through $\mathrm{VC}^{*}$ & 151.85 & 157.92 & 16.15 & 19.43 & 138.28 & 146.24 \\
\hline $\mathrm{F}_{3}: 50 \% \mathrm{RDF}+50 \% \mathrm{VC}$ & 143.79 & 151.58 & 12.01 & 14.12 & 128.19 & 136.54 \\
\hline $\mathrm{F}_{4}: 75 \% \mathrm{RDF}+25 \% \mathrm{VC}$ & 142.99 & 147.45 & 14.17 & 16.83 & 125.28 & 131.29 \\
\hline SEm \pm & 1.55 & 1.27 & 0.77 & 0.63 & 1.51 & 1.43 \\
\hline $\operatorname{LSD}(p=0.05)$ & 3.12 & 2.56 & 1.54 & 1.27 & 3.04 & 2.87 \\
\hline
\end{tabular}

*Vermicompost.

\section{Discussion}

Biomass production is a function of genetic character of the crop cultivar and the environmental factors, inputs applied and their management. Many factors are attributed to obtain the higher biomass production and among them, planting geometry plays a vital role in augmenting rice grain yield [7]. Wider spacing facilitates maximum light interception, better inter-culture operations and better soil aeration [8]. This could be reason for obtaining the maximum yield in wider row spacing.

Highest grain yield under inorganic sources of nutrients might be due to immediate release and availability of nutrients when compared with organic nutrients sources. Chemical fertilizers release nutrients instantly resulting higher crop biomass production. On the contrary, organic sources release nutrients slowly for longer period that does not meet the crop demand thus reduces crop biomass production. However, combined application of nutrients, $75 \% \mathrm{RD}$ through fertilizer and $25 \% \mathrm{~N}$ through VC, produced higher biomass due to synchronized and balanced nutrients supply for a longer period of time $[20,21]$. The yield advantage on the application of organic sources is due to their capability to supply essential nutrients other than N, P and K. Application of farm yard manure is known to increase concentrations of $\mathrm{Fe}, \mathrm{Mn}$, $\mathrm{Zn}$, and $\mathrm{Cu}$ in rice. Higher nutrients uptake with the application of inorganic fertilizer might be due to higher nutrient concentration along with higher biomass production [22,23]. Application of organic manure along with chemical fertilizer accelerates the microbial activity [24], increases nutrients use efficiency [25] and enhances the availability of the native nutrients to the plants resulting higher nutrients uptake [26]. Vermicompost applied plots built-up residual soil fertility because of slow release of nutrients and reduction of nutrient losses.

\section{Conclusions}

Rice cultivar, CNRH 3 produced maximum grain and straw yield. Rice, irrespective of cultivars, grown with inorganic fertilizers alone produced maximum grain and straw yield but it was statistically at par with that of application of $25 \%$ nutrient through organic and $75 \%$ through inorganic sources. Rice transplanted in $20 \mathrm{~cm} \times$ $20 \mathrm{~cm}$ spacing produced maximum biomass. Pro Agro 6201 rice cultivar, $25 \mathrm{~cm} \times 25 \mathrm{~cm}$ crop geometry, and sole organic manure had built-up maximum soil fertility. It may be recommend that rice cultivar CNRH 3, planting geometry $20 \mathrm{~cm} \times 20 \mathrm{~cm}$ and INM treatment $(25: 75$ 
organic: inorganic) can be adopted to obtain the higher biomass production while maintaining the soil fertility.

\section{REFERENCES}

[1] A. Jeyabal and G. Kuppuswamy, "Recycling of Organic Wastes for the Production of Vermicompost and Its Response in Rice-Legume Cropping System and Soil Fertility,” European Journal of Agronomy, Vol. 15, No. 3, 2001, pp. 153-170. doi:10.1016/S1161-0301(00)00100-3

[2] E. A. Siddiq, "Yawning Productivity Gaps," The Hindu Survey of Indian Agriculture, 2000, pp. 39-44.

[3] S. Peng, K. G. Cassman, S. S. Virmani, J. Sheehy and G. S. Khush, "Yield Potential Trends of Tropical Rice since Release of IR8 and the Challenge of Increasing Rice Yield Potential,” Crop Science, Vol. 39, No. 6, 1999, pp. 1552-1559. doi:10.2135/cropsci1999.3961552x

[4] E. A. Siddiq, "Rice Development Opportunities," The Hinhu Survey of Indian Agriculture, 1996, pp. 47-55.

[5] L. P. Yuan, "Hybrid Rice Breeding in China,” In: S. S. Virmani, et al., Eds., Advances in Hybrid Rice Technology, Proceeding of 3rd International Symposium on Hybrid Rice, Hyderabad, 14-16 November 1996, pp. 27-33.

[6] L. P. Yuan, "Hybrid Rice Breeding for Super High Yield," Hybrid Rice, Vol. 12, No. 6, 1999, pp. 1-6.

[7] R. Prasad, “A Textbook of Rice Agronomy,” Jain Brothers, New Delhi, 1999.

[8] A. K. Gautam, D. Kumar, Y. S. Shivay and B. N. Mishra, "Influence of Nitrogen Levels and Plant Spacing on Growth, Productivity and Quality of Two Inbred Varieties and a Hybrid of Aromatic Rice," Archives of Agronomy and Soil Science, Vol. 54, No. 5, 2008, pp. 515-532. doi: $10.1080 / 03650340802283470$

[9] R. B. Singh, "Environmental Consequences of Agricultural Development: A Case Study for the Green Revolution State of Haryana, India," Agriculture Ecosystem and Environment, Vol. 82, No. 1-3, 2000, pp. 97-103. doi:10.1016/S0167-8809(00)00219-X

[10] J. K. Friedel, "The Effect of Farming System on Labile Fractions of Organic Matter in Calcari-Epileptic Regosols,” Journal of Plant Nutrition Soil Science, Vol. 163, No. 1 , No. 2000, pp. 41-45. doi:10.1002/(SICI)1522-2624(200002)163:1<41::AID-JP LN41>3.0.CO;2-9

[11] S. K. Reddy, M. Singh, A. K. Tripathi, M. S. Singh and N. Manbendra, "Changes in Amount of Organic and Inorganic Fractions of Nitrogen in an Eutrochrept Soil after Long-Term Cropping with Different Fertilizer and Organic Manure Inputs," Journal of Plant Nutrient Soil Science, Vol. 166, No. 2, 2003, pp. 232-238.

[12] W. E. Larson and C. E. Clapp, "Effect of Organic Matter on Soil Physical Properties,” In: W. E. Larson and C. E. Clapp, Ed., Organic Matter and Rice, International Rice Research Institute, Manila, 1984, pp. 363-385.

[13] P. V. V. Prasad, V. Satyanarayana, V. R. K. Murthy and K. J. Boote, "Maximizing Yields in Rice-Groundnut Cropping Sequence through Integrated Nutrient Man- agement,” Field Crops Research, Vol. 75, No. 1, 2002, pp. 9-21. doi:10.1016/S0378-4290(01)00214-3

[14] A. Dobarmman and C. Witt, "The Potential Impact of Crop Intensification on Carbon and Nitrogen Cycling in Intensive Rice Systems,” In: G. J. D. Kirk and D. C. Olk, Eds., Carbon and Nitrogen Dynamics in Flooded Soils, International Rice Research Institute, Manila, 2000, pp. 1-25.

[15] D. S. Powlson and D. C. Olk, "Long-Term Soil Organic Matter Dynamics,” In: G. J. D. Kirk and D. C. Olk, Eds., Carbon and Nitrogen Dynamics in Flooded Soils, International Rice Research Institute, Manila, 2000, pp. 49-63.

[16] B. V. Subbiah and G. L. Asija, “A Rapid Procedure for Estimation of Available Nitrogen in Soil," Current Science, Vol. 25, No. 8, 1956, pp. 259-260.

[17] A. Walkley and I. A. Black, “An Examination of the Degtjareff Method for Determining Soil Organic Matter, and Proposed Modification of the Chromic Acid Titration Method,” Soil Science, Vol. 37, No. 1, 1934, pp. 29-38. doi:10.1097/00010694-193401000-00003

[18] S. R. Olsen, C. V. Cole, F. S. Watanabe and L. A. Dean, "Estimation of Available Phosphorus in Soil by Extraction with Sodium Bicarbonate,” US Department of Agriculture Circular No. 939, 1954.

[19] M. L. Jackson, “Soil Chemical Analysis,” Prentice Hall of India Pvt. Ltd, New Delhi, 1973.

[20] P. Pramanik, "Chemical and Biochemical Aspects of Vermicomposts with Reference to Transplanted Rice,” $\mathrm{PhD}$ Thesis, Visva Bharati University, Shantiniketan, 2007.

[21] M. Mwale, A. Mapiki and L. K. Phiri, “To Synchronize Nutrient Availability with Plant Uptake,” In: M. Mwale, A. Mapiki and L. K. Phiri, Ed., The Biology and Fertility of Tropical Soils, A TSBF Report 1997-1998, 2000, pp. 40-41.

[22] A. Swarup and N. P. S. Yaduvanshi, "Impact of Ten-Year Rice-Wheat Cropping System and integrated Nutrient Management on Soil Properties and Crop Productivity in a Gypsum Amended Sodic Soil," 18th World Congress of Soil Science, Philadelphia, 9-15 July 2006.

[23] P. Banik., P. K. Ghosal, T. K. Sasmal, S. Bhattacharya, B. K. Sarkar and D. K. Bagchi, "Effect of Organic and Inorganic Nutrients for Soil Quality Conservation and Yield of Rainfed Low Land Rice in Sub-Tropical Plateau Region," Journal of Agronomy and Crop Science, Vol. 192, No. 5, 2006, pp. 331-343. doi:10.1111/j.1439-037X.2006.00219.X

[24] R. Rani and O. P. Srivastava, "Vermicompost: A Potential Supplement to Nitrogenous Fertilizer in Rice Nutrition,” International Rice Research Notes, Vol. 22, No. 3, 1997, pp. 30-31.

[25] R. P. Narwal, M. Chaudhary, "Effect of Long-Term Application of FYM and Fertilizer N on Available P, K and S Content of Soil," 18th World Congress of Soil Science, Philadelphia, 9-15 July 2006.

[26] A. L. Bhandari, A. Sood, K. N. Sharma and D. S. Rana, "Integrated Nutrient Management in Rice-Wheat System,” Journal of Indian Society of Soil Science, Vol. 40, 1992, pp. 742-747. 